\title{
Ribonuclease-1 treatment after traumatic brain injury preserve the integrity of the neurovascular unit, decreases inflammatory response and delays secondary brain damage in mice
}

\section{Tobias J. Krämer}

University Medical Center of Johannes Gutenberg University Mainz

\section{Per Hübener}

University Medical Center of Johannes Gutenberg University Mainz

\section{Bruno Pöttker}

University Medical Center of Johannes Gutenberg University Mainz

\section{Christina Gölz}

University Medical Center of Johannes Gutenberg University Mainz

\section{Axel Neulen}

University Medical Center of Johannes Gutenberg University Mainz

\section{Tobias Pantel}

University Medical Center of Johannes Gutenberg University Mainz

\section{Hermann Goetz}

University Medical Center of Johannes Gutenberg University Mainz

\section{Katharina Ritter}

University Medical Center of Johannes Gutenberg University Mainz

\section{Serge C Thal ( $\nabla$ serge.thal@uni-wh.de )}

Helios University Hospital Wuppertal University Witten/Herdecke

\section{Michael K.E. Schäfer}

University Medical Center of Johannes Gutenberg University Mainz

\section{Research Article}

Keywords: brain injury, controlled-cortical-impact, cell-free RNA, RNase, mice, brain edema

Posted Date: October 14th, 2021

DOl: https://doi.org/10.21203/rs.3.rs-956514/v1

License: (c) (1) This work is licensed under a Creative Commons Attribution 4.0 International License. 
Page $2 / 19$ 


\section{Abstract}

Traumatic brain injury (TBI) involves primary mechanical damage and delayed secondary damage caused by vascular dysfunction and neuroinflammation. Intracellular components released into the parenchyma and systemic circulation, termed danger-associated molecular patterns (DAMPs), are major drivers of vascular dysfunction and neuroinflammation. These DAMPs include cell-free RNAs (cfRNAs), which damage the blood-brain barrier (BBB), thereby promoting edema, procoagulatory processes, and infiltration of inflammatory cells. We tested the hypothesis that intraperitoneal injection of Ribonuclease1 (RNase1, two doses of 20,60 , or $180 \mu \mathrm{g} / \mathrm{kg}$ ) at $30 \mathrm{~min}$ and $12 \mathrm{~h}$ after controlled-cortical-impact (CCl) can reduce secondary lesion expansion compared to vehicle treatment $24 \mathrm{~h}$ and $120 \mathrm{~h}$ post-CCI. The lowest total dose $(40 \mu \mathrm{g} / \mathrm{kg})$ was most effective at reducing lesion volume $(-31 \%$ RNase $40 \mu \mathrm{g} / \mathrm{kg}$ vs. vehicle), brain water accumulation $(-5.5 \%)$, and loss of BBB integrity $(-21.6 \%)$ at $24 \mathrm{~h}$ post-CCl. RNase1 also reduced perilesional leukocyte recruitment $(-53.3 \%)$ and microglial activation $(-18.3 \%)$ at $120 \mathrm{~h}$ post$\mathrm{CCl}$, but there was no difference in lesion volume at this time and no functional benefit. Treatment with RNase1 in the early phase following TBI stabilizes the BBB and impedes leukocyte immigration, thereby suppressing neuroinflammation. RNase1-treatment may be a novel approach to delay brain injury to extend the window for treatment opportunities after TBI.

\section{Introduction}

Mechanical stress to brain tissue induces necrosis and apoptosis (termed primary injury), resulting in the release of cellular contents into the brain parenchyma and blood circulation. ${ }^{1}$ Some intracellular biomolecules, including cell-free/extracellular ribonucleic acids (cfRNAs/eRNAs) act as damageassociated molecular patterns (DAMP) with potent pro-inflammatory activity. These cfRNAs are abundant in the extracellular milieu after traumatic brain injury (TBI), ${ }^{2}$ where they promote vascular endothelial grow factor (VEGF) activation and ensuing disruption of the blood blood-brain barrier (BBB), leading to edema and immune cell infiltration. ${ }^{3}$ These delayed processes are central drivers of the more expansive secondary damage following local TBI. ${ }^{4-6}$ Cell-free RNA has been reported to promote intercellular adhesion molecule-1 (ICAM-1)-dependent endothelial adhesion and extravasation of leukocytes. ${ }^{7}$ Further, sequence-specific cfRNA binding to toll-like receptors (TLRs, such as TLR7, 8, and 12) activates NF-KB pathway signaling and upregulates production of the pro-inflammatory cytokine tumor necrosis factor-a (TNF-a) by macrophages and microglia. ${ }^{8,9}$ Extracellular RNAs have also been shown to increase expression of cytokines by macrophages via TLR3, ${ }^{10,11}$ enhance interferon-y expression by astrocytes and microglia, ${ }^{12,13}$ support the maturation of dendritic cells, ${ }^{14}$ and exacerbate apoptosis via TLR3. ${ }^{15,16}$ Several animal studies that have examined the efficacy of immunomodulatory agents in improving outcomes after TBI have yielded positive results. ${ }^{17,18}$ Thus, elimination of cfRNAs at the site of injury or in the circulation could prevent secondary injury after TBI by stabilizing the BBB and promoting immunosuppression. ${ }^{19,20}$ 
The half-life of circulating cfRNA is dependent on plasma ribonuclease levels. ${ }^{21}$ Ribonuclease-1 (RNase1) is a member of the RNase-A superfamily expressed in exocrine pancreas, ${ }^{22}$ brain, ${ }^{23}$ and in vascular endothelial cells. ${ }^{24,25}$ The present study was designed to examine if RNase1 treatment post-TBI reduces brain edema, preserves BBB integrity, reduces brain lesion size, quells the neuroinflammatory response, and (or) improves functional outcome at 24 and $120 \mathrm{~h}$ after controlled-cortical-impact (CCl) in mice.

\section{Results}

Animals were randomly assigned to receive a low-dose of RNase1 (LD: $2 \times 20 \mu \mathrm{g} / \mathrm{kg}, \mathrm{n}=10$ ), an intermediate-dose of RNase1 (MD: $2 \times 60 \mu \mathrm{g} / \mathrm{kg}, \mathrm{n}=10$ ), a high-dose of RNase1 (HD: $2 \times 180 \mu \mathrm{g} / \mathrm{kg}, \mathrm{n}=$ $10)$, or vehicle $(2 \times 500 \mu \mathrm{L} \mathrm{NaCl} 0.9 \%, n=10)$ by i.p. injection 30 minutes and $12 \mathrm{~h}$ post-CCl. Contusion volume increase by $60 \%$ within $24 \mathrm{~h}$, indicating induction of secondary injury. ${ }^{26,27}$ However, all RNase 1 doses significantly reduced lesion volume compared to vehicle treatment (vehicle: $22.6 \pm 2.7 \mathrm{~mm}^{3}$; LD: $14.1 \pm 2.2 \mathrm{~mm}^{3} ; \mathrm{MD}: 16.3 \pm 2.9 \mathrm{~mm}^{3} ; \mathrm{HD}: 15.6 \pm 2.4 \mathrm{~mm}^{3}$; all $p<0.001$; Figure $1 \mathrm{~A}$ ). Lesion volumes continued to increase in all groups up to $120 \mathrm{~h}$ post-CCl and stabilized thereafter. Thus, post-CCl treatment with RNase1 can delay but not completely prevent brain damage (vehicle: $27.3 \pm 4.4 \mathrm{~mm}^{3}$; LD: $28.1 \pm 2.4 \mathrm{~mm}^{3}$; Figure 1B). Neurofunctional impairment was assessed by a deficit score ranging from 0 (no motor deficits) to 15 (severe impairment) according to the criteria of Timaru-Kast and colleagues. ${ }^{34}$ There were no significant differences in score among groups at $24 \mathrm{~h}$ post-CCl, although low-dose RNase 1 treatment resulted in an overall reduction compared to vehicle (4.5 \pm 1.3 vs. $5.9 \pm 1.9, p=0.0658$ ) (Figure $1 C$ ). There were no differences in motor function as assessed in the rotarod task at $120 \mathrm{~h}$ post- $\mathrm{CCl}$ (Figure 1D). To examine if RNase1 treatment suppresses neuroinflammation, mRNA expression levels of interleukin (IL)-1 $\beta$ (Figure 2A) and IL-6 (Figure 2B) were determined in brain samples from the lesioned (ipsilateral) hemisphere by qPCR at $24 \mathrm{~h}$ post-CCl, but no differences were found among treatment groups. In addition, the number of activated microglia in the ipsilateral hemisphere as indicated by Iba-1 immunostaining was elevated in all treatment groups compared to the corresponding contralateral hemisphere and did not differ among groups at $24 \mathrm{~h}$ post-CCI (Figure $2 \mathrm{C}$ ). In contrast, cerebral inflammation was enhanced in the ipsilateral hemisphere at $120 \mathrm{~h}$ post-CCl, and both the numbers of Iba$1+$ and CD 45+ cells were significantly reduced by RNase 1 treatment (Iba- $1+, p=0.029 ; \mathrm{CD} 45+, p=0.01$ ) (Figure 2D, E).

All subsequent experiments compared vehicle or sham controls to a LD RNase1 treatment group ( $n=10$ mice per group) to reduce the number of animals. First, the influence of LD RNase1 on post-CCI brain edema was examined by comparing the change in brain weight following vacuum drying (an estimate of water content). Treatment with RNase significantly reduced post-CCl brain water content from $81.6 \% \pm$ $2.3 \%$ (contralateral: $74.9 \% \pm 1.1 \%$ ) to $77.1 \% \pm 2.0 \%, p=0.0027$ (contralateral: $72.1 \% \pm 2.3 \%$ ), suggesting prevention of post-CCl edema. However, post-CCl water content was still significantly higher than contralateral or in the sham group (ipsilateral: $72.3 \% \pm 2.3 \% ; p<0.001$; contralateral: $73.6 \% \pm 2.0$; Figure $3 A$ ), indicating only partial efficacy. To determine if LD RNase1 treatment also stabilizes BBB integrity, IgG extravasation was quantified as a surrogate parameter by dot blotting. These measurements revealed a 
2-3-fold higher signal in post-CCl tissue compared to sham operated animals (Figure 3B) and a significant reduction by RNase 1 treatment ( $40 \%$ lower IgG extravasation, $p=0.0073$ ) compared to vehicle. To maintain BBB integrity, adequate structural support from tight-junction (TJ) proteins is essential. We therefore quantified the expression levels of mRNAs encoding the TJ proteins claudin-5, ZO1 , and ZO-2 by qPCR at $24 \mathrm{~h}$ post-CCl. mRNA expression levels of all measured TJ proteins were significantly higher in the LD RNase1 group $(+140-160 \%)$ compared to vehicle-treated controls (Figure 3C, D, E).

\section{Discussion}

This is the first study demonstrating that RNase 1 injection can preserve the integrity of the neurovascular unit in the early period following TBI. Low-dose RNase1 upregulated multiple TJ protein mRNA levels, and reduced edema, inflammatory cell infiltration, macrophage activation and lesion volume. However, the treatment protocol ( 2 doses at $30 \mathrm{~min}$ and $12 \mathrm{~h}$ post-contusion) was not sufficient for long-term reduction in lesion size, suggesting that pretreatment or prolonged post-treatment may be necessary. For instance, a protective effect against postoperative dementia and brain edema after stroke could only be achieved with RNase pretreatment. ${ }^{19,28}$

\section{Effects On Brain Edema}

Vasogenic edema and IgG extravasation were reduced at one day post-TBI. Under cerebral ischemia, vascular permeability is increased by cfRNA via the VEGF axis. ${ }^{3}$ TBI leads to an upregulation ${ }^{29}$ or downregulation ${ }^{30}$ of VEGF and would be a possible candidate for more detailed analyses in future studies. The pathogenic effect of cfRNA may be partially attributed to reduced ZO-1 and claudin-5

expression, ${ }^{31}$ while RNase 1 can increase expression of ZO-1, ZO-2, and claudin-5, suggesting a stabilizing effect on BBB integrity.

\section{Anti-inflammatory Effects}

In addition to VEGF signaling, cfRNAs stimulate the immune system via TLRs, ${ }^{8,9,15,32}$ which induces the release of pro-inflammatory cytokines ${ }^{7}$ and shifts microglial phenotype to the pro-inflammatory M1 type 33,34 known to induce neuronal damage and CNS dysfunction. ${ }^{35}$ However, these anti-inflammatory actions appear to play only a minor role in the first $24 \mathrm{~h}$ post-CCl as there no alterations in IL- 6 and IL-1 $\beta$ mRNA expression or microglial number, while significantly reduced numbers of Iba $1+$ and CD $45+$ cells were found at $120 \mathrm{~h}$ post-CCl. Due to the early beneficial effects on the BBB and immune system, ${ }^{7-16}$ reduced neuroinflammation was still detected $120 \mathrm{~h}$ post-TBI. Collectively, these findings suggest that cfRNAs released during TBI induce leukocyte extravasation via VEGF2 activation ${ }^{7}$ and a simultaneous shift to the pro-inflammatory M1-phenotype. ${ }^{12}$ 
At 5 days after injury, brain lesion volume was similar between groups, suggesting that the initial putative beneficial effect was not long lasting and not sufficient to results in functional improvement.

\section{Limitations Of The Study}

These findings suggest that RNase 1 treatment may be a useful adjunct therapy to reduce or delay secondary damage in the early phase post-TBI. Indeed, high cfRNA in systemic circulation is associated with poor outcome after TBI. ${ }^{20,36}$ Further, RNase1 released from endothelial cells during ischemia 25,37 may serve an endogenous protective function. ${ }^{38}$ Thus, RNase1 treatment supplements an endogenous mechanism. RNase1 degrades ssRNAs but not extracellular rRNAs, mRNAs, or miRNAs. In a mouse model of myocardial infarction, RNAse1 treatment reduced myocardial edema and infarct size. ${ }^{38,39}$ While this presumably reflects degradation of cfRNA, it is currently challenging to directly measure cfRNAs in small volumes of mouse plasma. ${ }^{28,39,40}$ However, these protective effects of RNase1 treatment after TBI are consistent with suppression of pathways known to be activated by cfRNAs. RNase1 attenuated septic cardiomyopathy and cardiac apoptosis in a murine model of polymicrobial sepsis. Whereas there was no clear evidence of reduction in cfRNA nor a correlation between RNase1 and cfRNA levels. ${ }^{40}$ After myocardial infarction in mice systemic application of RNase1 reduced edema formation and infarct size and improved survival. Plasma cfRNA levels were significantly increased compared with controls at 30 minutes after ligation, but no difference were present between treatment groups. ${ }^{39}$ Exosomes $^{41}$, microRNA ${ }^{42}$, or long non-coding RNA ${ }^{43}$ could possibly serve as indirect markers for cfRNA. Especially in in vivo studies with small rodents to overcome the limitation of limited plasma sample size.

Despite clear data on brain edema formation and early histological brain damage, the present study fails to provide evidence for functional improvement by RNase 1 therapy after experimental TBI. Although the RNase1-treated animals have lower mean scores in the neuro-deficit score $24 \mathrm{~h}$ after $\mathrm{CCl}$, these changes were not significant. Rotarod data failed to show any effect at $24 \mathrm{~h}$ and 5 days after injury. Reduction in extent of brain edema, blood-brain-barrier disruption and brain lesion at 24 hours post injury therefore does not result in improved functional recovery. Furthermore, brain water levels were lower than expected and compared to recent other studies. Although the vacuum method is superior to the conventional oven technique in terms of quantifying water content in small samples, ${ }^{44}$ the low water levels in all samples suggest a systemic measurement bias, which was equally evident in all experimental groups. Therefore, we decided to present the ipsi- and contralateral data to demonstrate the relative changes induced by RNase1 treatment.

In addition, only male animals are used in the study. We therefore cannot estimate the gender effect and the impact of RNase 1 treatment in brain injured female mice. These effects need to be addressed in future studies.

\section{Conclusion}


We demonstrate that i.p. RNase1 can stabilize the BBB following $\mathrm{TBI}$, resulting in reduced vasogenic edema, leukocyte recruitment, and microglial activation in perilesional tissue. These data support a role for cfRNA and RNase1 in pathophysiology of TBI.

\section{Materials And Methods}

\section{Animals}

This study was approved by the German animal protection committee (Landesuntersuchungsamt Rheinland-Pfalz, protocol number G-13-1-074). Experiments were performed in accordance with all national animal welfare guidelines and ARRIVE guidelines for reporting animal research. ${ }^{45}$ The study was conducted using 90 male C57BI6/N mice (Charles River Laboratories, Sulzfeld, Germany; weight: 22-28 g). Two mice had to be excluded from the study before the end of the trial.

\section{Drug preparation}

Ribonuclease-1 (RNase1, Sigma, Germany) was dissolved in sterile normal saline by a third person not involved in the experiments or analysis, numbered and prepared for intraperitoneal (i.p.) injection. Mice were randomly allocated to receive either one or two i.p. injections of 20,60 , or $180 \mu \mathrm{g} / \mathrm{kg} \mathrm{RNase} 1$ or vehicle at $30 \mathrm{~min}$ and $12 \mathrm{~h}$ post-TBI. The experiments and the processing of the material was performed in blinded fashion. These doses are at least tenfold lower than required to induce adverse side effects in rodents. $3,19,28$ To minimize the total number of animals used in accordance with ARRIVE guidelines ${ }^{45}$, no additional sham group was established for most experiments. Also, after the initial dose-response study, subsequent investigations of brain water accumulation, tissue IgG changes, and 120-h survival used only the most effective (low) dose of RNase1, with sham animals examined for comparison only when necessary.

\section{Traumatic brain injury}

Traumatic brain injury was induced by $\mathrm{CCl}$ using an electromagnetic impact device (Leica Impact One ${ }^{\mathrm{TM}}$ Stereotaxic Impactor, Richmond, IL; tip: 3 mm; velocity: 6 m/s; duration: 200 ms; displacement: $1.5 \mathrm{~mm}$ ). Animals were anesthetized by intraperitoneal injection of $5 \mathrm{mg} / \mathrm{kg}$ midazolam, $0.05 \mathrm{mg} / \mathrm{kg}$ fentanyl, and $0.5 \mathrm{mg} / \mathrm{kg}$ medetomidine, and body temperature controlled at $37^{\circ} \mathrm{C}$ based on feedback from a rectal thermometer. After craniotomy, trauma was induced on the right cortex by an experimenter blinded to treatment group allocation. ${ }^{46-48}$ Thereafter, the craniotomy was closed and anesthesia was antagonized using $0.5 \mathrm{mg} / \mathrm{kg}$ Flumazenil and $2.5 \mathrm{mg} / \mathrm{kg}$ Atepamezol hydrochloride. Animals were allowed to recover for $1.5 \mathrm{~h}$ in a neonatal incubator (IC8000, Draeger, Luebeck, Germany) with controlled air temperature $\left(35^{\circ} \mathrm{C}\right)$ and ambient humidity $(35 \%)$.

\section{Neurological outcome and Rotarod test}

Before $\mathrm{CCl}(1 \mathrm{~h})$ and at $24 \mathrm{~h}$ after $\mathrm{CCl}$, an investigator blinded to experimental group assigned a neurologic severity score ranging from 0 (healthy) to 15 (severely impaired) (24 h groups: $N=40) .{ }^{49} \mathrm{An}$ 
accelerating rotarod test (Rotarod Treadmill, MED Associates, INC, St Albans, VT) was conducted in the long-time surviving groups one day before and at 24 and $120 \mathrm{~h}$ post-CCI $(\mathrm{N}=20)$. Briefly, each mouse was placed on an accelerating rotating cylinder, and the time and maximum speed at which the animal fell off ( $27 \mathrm{~cm}$ fall height) were recorded. ${ }^{50,51}$ Briefly, four rotarod tasks were conducted before $\mathrm{CCl}$ and the mean value of each animal was defined as $100 \%$ "starting value". $24 \mathrm{~h}$ and $120 \mathrm{~h}$ following injury, animals were tested in two trials per time point, averaged and evaluated relative to pre-injury latencies to correct for individual pre-injury performance.

\section{Tissue preparation, Nissl, Iba-1 and CD45 staining}

Animals were anesthetized and brains excised, frozen in powdered dry ice, and stored at $-20^{\circ} \mathrm{C}$. Coronal sections (10- $\mu \mathrm{m}$ thick) were cut at 500- $\mu \mathrm{m}$ intervals ( $24 \mathrm{~h}: \mathrm{n}=40$ mice, $120 \mathrm{~h}: \mathrm{n}=20$ mice) and subjected to cresyl violet staining. Brain lesion volume was measured using a computerized image system (DeltaPix InSight, Smorum, Denmark). ${ }^{52}$ In brief, to control for the effect of brain edema, the area of uninjured brain tissue and the total area of the contralateral hemisphere were quantified in each section. Afterwards the injured area was calculated by subtraction of "normal" area in the injured hemisphere from total contralateral. Other slices were immunostained for the activated microglial marker Iba- 1 using an anti-rabbit Iba-1 antibody (1:1500, Wako Pure Chemical Industries, Osaka, Japan) and a biotinylated anti-rabbit IgG (Vector Laboratories Inc., Burlingame, CA). Signals were detected using ABC Complex (Vector) and DAB (Thermo-Fischer, Waltham, MA). The appearance of Iba- $1+/ C D 45+$ cells $120 \mathrm{~h}$ after lesion induction was quantified by dual staining using rat anti-CD45 (1:500, Thermo-Fisher) with Alexa Fluor 568-conjugated goat anti-rat IgG (1:500, Thermo-Fisher, Waltham, MA) and anti-rabbit Iba-1 antibody (1:500, Wako) with Alexa Fluor 488-conjugated goat anti-rabbit lgG (1:500, Thermo-Fisher). Sections were then counterstained with DAPI (1:10000, Thermo-Fisher). ${ }^{53}$ Images were acquired at 20x magnification, and cells were counted in the perilesional and corresponding, non-injured contralateral regions of two serial sections in the zone with the largest lesion (bregma $-1.64 \mathrm{~mm}$ and bregma $-1.82 \mathrm{~mm}$, www.mbl.org, ROI: $2.55 \mathrm{~mm}^{2}$ ). ${ }^{51} \mathrm{In}$ the predefined areas (border zone at lower outside corner the lesion and the corresponding contralateral side) double-immunolabeled cells were counted by an investigator blinded to treatment using ImageJ (U.S. National Institutes of Health, Bethesda, MD).

\section{RNA isolation and quantitative polymerase chain reaction (qPCR)}

Samples from the contused brain region were collected $(n=40)$ as described, ${ }^{22}$ frozen in liquid nitrogen, and stored at $-80^{\circ} \mathrm{C}$. RNA extraction, reverse transcription, and mRNA quantification by real-time RT-PCR were performed as described. ${ }^{49,52}$ Absolute copy numbers of target genes were normalized to the reference gene cyclophilin $\mathrm{A}(P P I A)$.

\section{Brain water content}

Brains were isolated $24 \mathrm{~h}$ after lesion and the hemispheres cut along the interhemispheric plane $(\mathrm{n}=30)$. The hemispheres were weighed (wet weight, WW), dried in a vacuum-centrifuge (Univapo 100H, UniEquip, 
Planegg, Germany) for $48 \mathrm{~h}$, and reweighed (dry weight, DW). Brain water content was calculated according to the equation water content $(\%)=(W W-D W) / W W \times 100.44$

\section{IgG quantification}

Brain tissue samples $(n=30$ mice) were lysed in RIPA buffer, and total cellular proteins separated by SDSPAGE, transferred to nitrocellulose membranes, immunolabeled (IgG; $1: 10$ 000; Li-cor), and visualized by a near-infrared laser scanning system (LI-COR Odyssey) essentially as described. ${ }^{54} \mathrm{An}$ antibody against GAPDH (Acris, clone 6C5) was used as the reference and quantification was performed using ImageJ $(\mathrm{NIH}, \mathrm{MD})$.

\section{Statistics}

All experiments were randomized and performed by blinded investigators (computer-based randomization). The experimental groups are presented in Figure 4. To determine the required sample size, an a priori power analysis was performed using $\mathrm{G} *$ Power $^{55}$ based on lesion volume data from previous studies. A priori power analysis for an effect size of 0.7 suggested that a standard statistical power (1- $\beta$ ) of $P \beta=0.95$ and significance level (a) of 0.05 can be obtained for expected lesion volumes using 10 subjects per group (4 groups) and for expected brain water content using 10 subjects per group (2 groups). GraphPad Prism 9 statistical software (GraphPad Software Inc., La Jolla, CA, USA) was used to perform statistical analysis. Prior to analysis, we checked the test assumptions. Due to the limited power in small samples, we did not perform formal goodness-of-fit tests prior to the $t$ test or ANOVA, but instead relied on the graphical assessment of distribution characteristics. ${ }^{56}$ Normality was checked by inspecting the unimodality and symmetry of histograms, as well as by Q-Q plots. The equality of variances was checked by inspecting histograms and standard deviations. For comparison of multiple independent groups, Brown-Forsythe and Welch ANOVA with post-hoc Dunnett T3 multiple comparisons test (comparisons between all groups) was employed. To evaluate group differences in repeatedmeasurements from the same animals (rotarod), RM two-way ANOVA was applied (factors: treatment and time), followed by Šidáks multiple comparisons test. Comparisons between two independent groups were carried out by the Welch's $t$ test. Values of $p<0.05$ were considered significant. Data are presented as the mean and standard deviation (mean $\pm \mathrm{SD}$ ).

\section{Declarations}

\section{Acknowledgments}

The authors thank Wieslawa Bobkiewicz, Tobias Hirnet, Frida Kornes, Dana Pieter, and Florian Pickart for their skillful assistance and critical validation during experimental investigations.

\section{Authors' contributions}

TK: conception and design, experimental procedures, analysis and interpretation of the results, writing the manuscript. PH: experimental procedures, analysis of the results. BP, CG, HG, AN, TP: analysis of the 
results, critical revision of the manuscript. KR, MS: immunhistochemistry, interpretation of the results, critical revision of the manuscript. ST: conception and design, analysis and interpretation of the results, writing the manuscript.

\section{Author disclosure statement}

The authors declare no conflicting financial interests. Some data presented in this manuscript are part of the doctoral thesis of $\mathrm{PH}$ and of the professorial dissertation (Habilitation) of TK.

\section{Funding statement}

This work was supported by institutional funding.

\section{Data Sharing}

The data that support the findings of this study are available from the corresponding author, SCT, upon reasonable request.

\section{References}

1. Pan, Y. B., Sun, Z. L. \& Feng, D. F. The Role of MicroRNA in Traumatic Brain Injury., 367, 189-199 https://doi.org/10.1016/j.neuroscience.2017.10.046 (2017).

2. Vourc'h, M., Roquilly, A. \& Asehnoune, K. Trauma-Induced Damage-Associated Molecular PatternsMediated Remote Organ Injury and Immunosuppression in the Acutely III Patient. Frontiers in immunology, 9, 1330 https://doi.org/10.3389/fimmu.2018.01330 (2018).

3. Fischer, S. et al. Extracellular RNA mediates endothelial-cell permeability via vascular endothelial growth factor., 110, 2457-2465 https://doi.org/10.1182/blood-2006-08-040691 (2007).

4. Werner, C. \& Engelhard, K. Pathophysiology of traumatic brain injury. British journal of anaesthesia, 99, 4-9 https://doi.org/10.1093/bja/aem131 (2007).

5. Thal, S. C. \& Neuhaus, W. The blood-brain barrier as a target in traumatic brain injury treatment. Arch Med Res, 45, 698-710 https://doi.org/10.1016/j.arcmed.2014.11.006 (2014).

6. Toffolo, K. et al. Circulating microRNAs as biomarkers in traumatic brain injury., 145, 199-208 https://doi.org/10.1016/j.neuropharm.2018.08.028 (2019).

7. Fischer, S. et al. Extracellular RNA promotes leukocyte recruitment in the vascular system by mobilising proinflammatory cytokines. Thromb Haemost, 108, 730-741 https://doi.org/10.1160/TH12-03-0186 (2012).

8. Bertheloot, D. et al. RAGE Enhances TLR Responses through Binding and Internalization of RNA. Journal of immunology (Baltimore, Md.: 1950) 197, 4118-4126, doi:10.4049/jimmunol.1502169 (2016).

9. Gantier, M. P. et al. TLR7 Is Involved in Sequence-Specific Sensing of Single-Stranded RNAs in Human Macrophages. The Journal of Immunology, 180, 2117-2124 
https://doi.org/10.4049/jimmunol.180.4.2117 (2008).

10. Noll, F. et al. Self-extracellular RNA acts in synergy with exogenous danger signals to promote inflammation. PLoS One, 12, e0190002 https://doi.org/10.1371/journal.pone.0190002 (2017).

11. Alexopoulou, L., Holt, A. C., Medzhitov, R. \& Flavell, R. A. Recognition of double-stranded RNA and activation of NF-kappaB by Toll-like receptor 3. Nature, 413, 732-738 https://doi.org/10.1038/35099560 (2001).

12. Melton, L. M. et al. Chronic glial activation, neurodegeneration, and APP immunoreactive deposits following acute administration of double-stranded RNA. Glia, 44, 1-12 https://doi.org/10.1002/glia.10276 (2003).

13. Jensen, M. B., Hegelund, I. V., Lomholt, N. D., Finsen, B. \& Owens, T. IFNgamma enhances microglial reactions to hippocampal axonal degeneration. The Journal of neuroscience: the official journal of the Society for Neuroscience, 20, 3612-3621 (2000).

14. Zhang, L. J. et al. Antimicrobial Peptide LL37 and MAVS Signaling Drive Interferon-beta Production by Epidermal Keratinocytes during Skin Injury., 45, 119-130 https://doi.org/10.1016/j.immuni.2016.06.021 (2016).

15. Chen, C. et al. Role of extracellular RNA and TLR3-Trif signaling in myocardial ischemia-reperfusion injury. Journal of the American Heart Association, 3, e000683 https://doi.org/10.1161/jaha.113.000683 (2014).

16. Kim, H. et al. Double-stranded RNA mediates interferon regulatory factor 3 activation and interleukin6 production by engaging Toll-like receptor 3 in human brain astrocytes., 124, 480-488 https://doi.org/10.1111/j.1365-2567.2007.02799.x (2008).

17. Begemann, M., Leon, M., van der Horn, H. J., van der Naalt, J. \& Sommer, I. Drugs with antiinflammatory effects to improve outcome of traumatic brain injury: a meta-analysis. Scientific reports, 10, 16179 https://doi.org/10.1038/s41598-020-73227-5 (2020).

18. Tang, H. et al. Progesterone and vitamin D: Improvement after traumatic brain injury in middle-aged rats. Hormones and behavior, 64, 527-538 https://doi.org/10.1016/j.yhbeh.2013.06.009 (2013).

19. Walberer, M. et al. RNase therapy assessed by magnetic resonance imaging reduces cerebral edema and infarction size in acute stroke. Curr Neurovasc Res, 6, 12-19 (2009).

20. Macher, H. et al. Role of early cell-free DNA levels decrease as a predictive marker of fatal outcome after severe traumatic brain injury. Clin Chim Acta, 414, 12-17 https://doi.org/10.1016/j.cca.2012.08.001 (2012).

21. Futami, J. et al. Tissue-specific expression of pancreatic-type RNases and RNase inhibitor in humans. DNA and cell biology, 16, 413-419 https://doi.org/10.1089/dna.1997.16.413 (1997).

22. Morita, T., Niwata, Y., Ohgi, K., Ogawa, M. \& Irie, M. Distribution of two urinary ribonuclease-like enzymes in human organs and body fluids. Journal of biochemistry, 99, 17-25 (1986).

23. Yasuda, T., Nadano, D., Takeshita, H. \& Kishi, K. Two distinct secretory ribonucleases from human cerebrum: purification, characterization and relationships to other ribonucleases. The Biochemical journal, 296 (Pt 3), 617-625 (1993). 
24. Barrabes, S. et al. Glycosylation of serum ribonuclease 1 indicates a major endothelial origin and reveals an increase in core fucosylation in pancreatic cancer., 17, 388-400 https://doi.org/10.1093/glycob/cwm002 (2007).

25. Landre, J. B. et al. Human endothelial cells selectively express large amounts of pancreatic-type ribonuclease (RNase 1). J Cell Biochem, 86, 540-552 https://doi.org/10.1002/jcb.10234 (2002).

26. Timaru-Kast, R. et al. Delayed inhibition of angiotensin II receptor type 1 reduces secondary brain damage and improves functional recovery after experimental brain trauma*. Critical care medicine, 40, 935-944 https://doi.org/10.1097/CCM.0b013e31822f08b9 (2012).

27. Thal, S. C. et al. Pioglitazone reduces secondary brain damage after experimental brain trauma by PPAR-gamma-independent mechanisms. Journal of neurotrauma, 28, 983-993 https://doi.org/10.1089/neu.2010.1685 (2011).

28. Chen, C. et al. Protective Effect of RNase on Unilateral Nephrectomy-Induced Postoperative Cognitive Dysfunction in Aged Mice. PLoS One, 10, e0134307 https://doi.org/10.1371/journal.pone.0134307 (2015).

29. Sköld, M. K., von Gertten, C., Sandberg-Nordqvist, A. C., Mathiesen, T. \& Holmin, S. VEGF and VEGF receptor expression after experimental brain contusion in rat. Journal of neurotrauma, 22, 353-367 https://doi.org/10.1089/neu.2005.22.353 (2005).

30. Wright, D. K. et al. Behavioral, blood, and magnetic resonance imaging biomarkers of experimental mild traumatic brain injury. Scientific reports, 6, 28713 https://doi.org/10.1038/srep28713 (2016).

31. Thal, S. C. et al. Volatile anesthetics influence blood-brain barrier integrity by modulation of tight junction protein expression in traumatic brain injury. PLoS One, 7, e50752 https://doi.org/10.1371/journal.pone.0050752 (2012).

32. Ganguly, D. et al. Self-RNA-antimicrobial peptide complexes activate human dendritic cells through TLR7 and TLR8. J Exp Med, 206, 1983-1994 https://doi.org/10.1084/jem.20090480 (2009).

33. Cabrera-Fuentes, H. A. et al. Regulation of monocyte/macrophage polarisation by extracellular RNA. Thromb Haemost, 113, 473-481 https://doi.org/10.1160/TH14-06-0507 (2015).

34. Kumar, A. et al. Microglial-derived microparticles mediate neuroinflammation after traumatic brain injury. J Neuroinflammation, 14, 47 https://doi.org/10.1186/s12974-017-0819-4 (2017).

35. Stoica, B. A. \& Faden, A. I. Cell death mechanisms and modulation in traumatic brain injury., 7, 3-12 https://doi.org/10.1016/j.nurt.2009.10.023 (2010).

36. Rodrigues Filho, E. M. et al. Elevated cell-free plasma DNA level as an independent predictor of mortality in patients with severe traumatic brain injury. Journal of neurotrauma, 31, 1639-1646 https://doi.org/10.1089/neu.2013.3178 (2014).

37. Sznajd, J., Magdon, M., Naskalski, J. W., Uracz, R. \& Wojcikiewicz, O. Serum ribonuclease activity in acute myocardial infarction. Cor et vasa, 23, 241-247 (1981).

38. Cabrera-Fuentes, H. A. et al. RNase1 prevents the damaging interplay between extracellular RNA and tumour necrosis factor-alpha in cardiac ischaemia/reperfusion injury. Thromb Haemost, 112, 11101119 https://doi.org/10.1160/th14-08-0703 (2014). 
39. Stieger, P. et al. Targeting of Extracellular RNA Reduces Edema Formation and Infarct Size and Improves Survival After Myocardial Infarction in Mice. J Am Heart Assoc, 6, https://doi.org/10.1161/JAHA.116.004541 (2017).

40. Zechendorf, E. et al. Ribonuclease 1 attenuates septic cardiomyopathy and cardiac apoptosis in a murine model of polymicrobial sepsis. JCl insight, https://doi.org/10.1172/jci.insight.131571 (2020).

41. Wang, P. et al. Plasma Exosome-derived MicroRNAs as Novel Biomarkers of Traumatic Brain Injury in Rats. International journal of medical sciences, 17, 437-448 https://doi.org/10.7150/ijms.39667 (2020).

42. Atif, H. \& Hicks, S. D. A Review of MicroRNA Biomarkers in Traumatic Brain Injury. Journal of experimental neuroscience, 13, 1179069519832286 https://doi.org/10.1177/1179069519832286 (2019).

43. Cuevas-Diaz Duran, R., Wei, H., Kim, D. H. \& Wu, J. Q. Invited Review: Long non-coding RNAs: important regulators in the development, function and disorders of the central nervous system. Neuropathology and applied neurobiology, 45, 538-556 https://doi.org/10.1111/nan.12541 (2019).

44. Sebastiani, A., Hirnet, T., Jahn-Eimermacher, A. \& Thal, S. C. Comparison of speed-vacuum method and heat-drying method to measure brain water content of small brain samples. J Neurosci Methods, 276, 73-78 https://doi.org/10.1016/j.jneumeth.2016.11.012 (2017).

45. Kilkenny, C., Browne, W. J., Cuthill, I. C., Emerson, M. \& Altman, D. G. Improving bioscience research reporting: The ARRIVE guidelines for reporting animal research. Journal of pharmacology \& pharmacotherapeutics, 1, 94-99 https://doi.org/10.4103/0976-500x.72351 (2010).

46. Thal, S. C., Wyschkon, S., Pieter, D., Engelhard, K. \& Werner, C. Selection of endogenous control genes for normalization of gene expression analysis after experimental brain trauma in mice. $J$ Neurotrauma, 25, 785-794 https://doi.org/10.1089/neu.2007.0497 (2008).

47. Brody, D. L. et al. Electromagnetic controlled cortical impact device for precise, graded experimental traumatic brain injury. Journal of neurotrauma, 24, 657-673 https://doi.org/10.1089/neu.2006.0011 (2007).

48. Kramer, T. et al. Dimethyl fumarate treatment after traumatic brain injury prevents depletion of antioxidative brain glutathione and confers neuroprotection. Journal of neurochemistry, 143, 523533 https://doi.org/10.1111/jnc.14220 (2017).

49. Timaru-Kast, R. et al. Influence of age on brain edema formation, secondary brain damage and inflammatory response after brain trauma in mice. PLoS One, 7, e43829 https://doi.org/10.1371/journal.pone.0043829 (2012).

50. Pottker, B. et al. Traumatic brain injury causes long-term behavioral changes related to region-specific increases of cerebral blood flow. Brain structure \& function, 222, 4005-4021 https://doi.org/10.1007/s00429-017-1452-9 (2017).

51. Kramer, T. J. et al. Depletion of regulatory $T$ cells increases $T$ cell brain infiltration, reactive astrogliosis, and interferon-gamma gene expression in acute experimental traumatic brain injury. $J$ Neuroinflammation, 16, 163 https://doi.org/10.1186/s12974-019-1550-0 (2019). 
52. Sebastiani, A. et al. Proneurotrophin Binding to P75 Neurotrophin Receptor (P75ntr) Is Essential for Brain Lesion Formation and Functional Impairment after Experimental Traumatic Brain Injury. $J$ Neurotrauma, 32, 1599-1607 https://doi.org/10.1089/neu.2014.3751 (2015).

53. Schaible, E. V. et al. 2-Methoxyestradiol confers neuroprotection and inhibits a maladaptive HIF1 alpha response after traumatic brain injury in mice. J Neurochem, 129, 940-954 https://doi.org/10.1111/jnc.12708 (2014).

54. Menzel, L. et al. Progranulin protects against exaggerated axonal injury and astrogliosis following traumatic brain injury. Glia, 65, 278-292 https://doi.org/10.1002/glia.23091 (2017).

55. Faul, F., Erdfelder, E., Buchner, A. \& Lang, A. G. Statistical power analyses using G*Power 3.1: tests for correlation and regression analyses. Behav Res Methods, 41, 1149-1160 https://doi.org/10.3758/BRM.41.4.1149 (2009).

56. Schucany, W. R. \& Tony Ng, H. K. Preliminary Goodness-of-Fit Tests for Normality do not Validate the One-Sample Student t. Communications in Statistics - Theory and Methods, 35, 2275-2286 https://doi.org/10.1080/03610920600853308 (2006).

\section{Figures}


A Influence on histological brain damage (24 h)

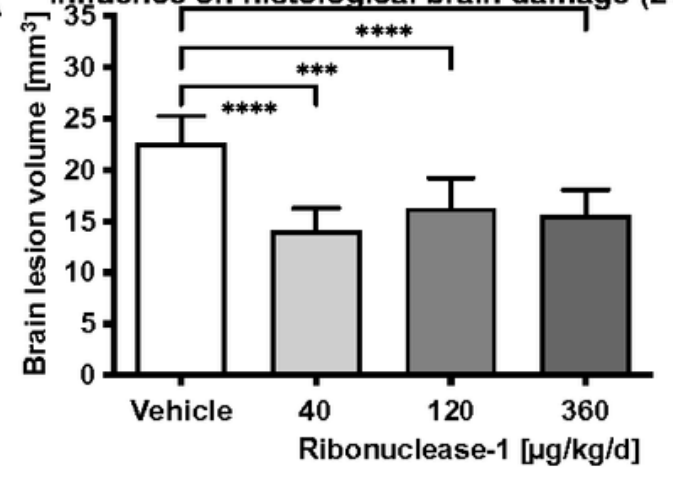

B

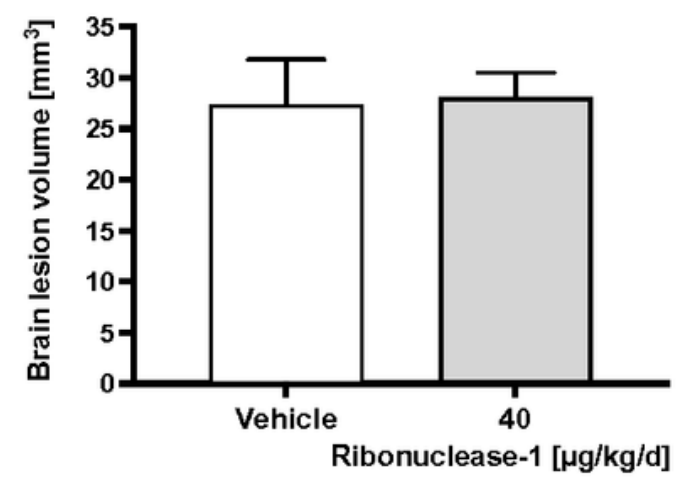

C Influence on neurofunctional outcome (24 h)

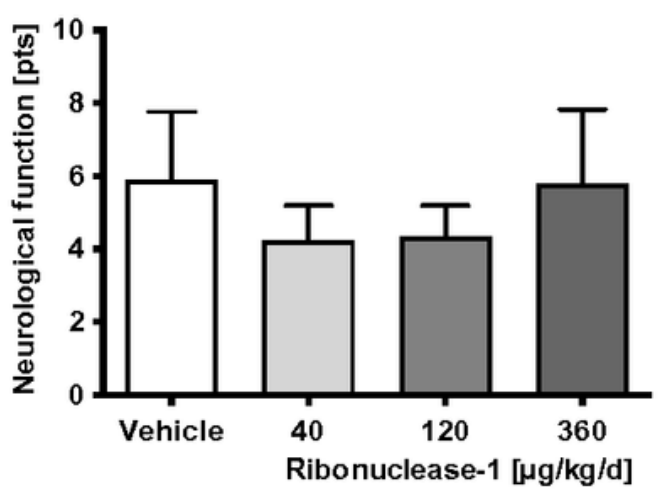

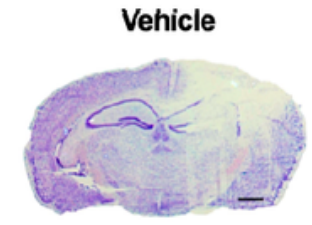

$120 \mu \mathrm{g} / \mathrm{kg} / \mathrm{d}$
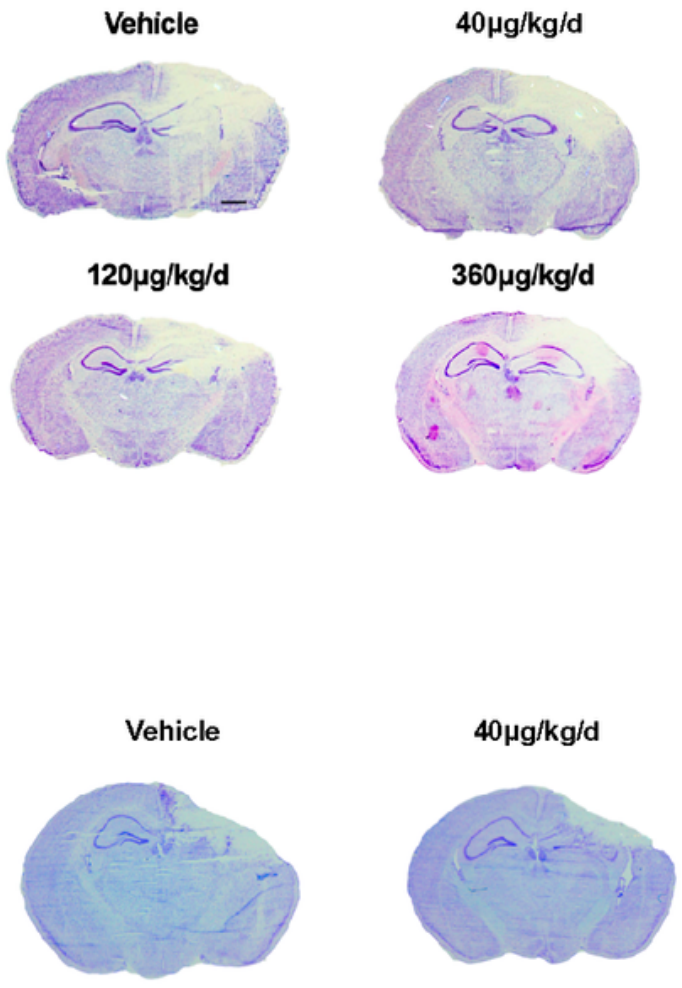

D

Influence on motor function $(120 \mathrm{~h})$

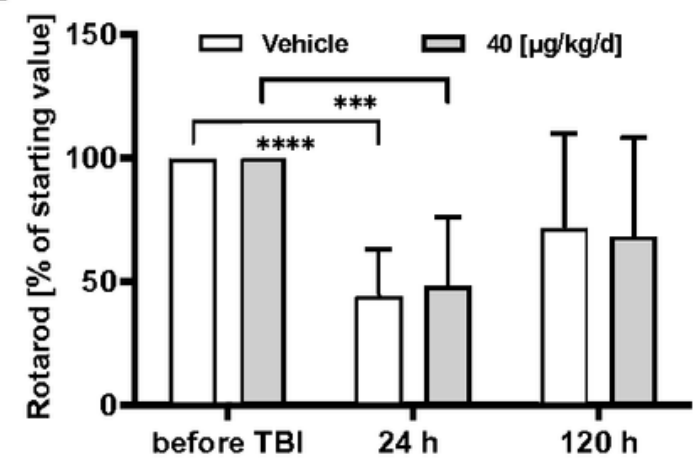

Figure 1

Influence on brain lesion and functional outcome Brain lesion was determined 24 hours (A) after controlled cortical impact injury in animals treated with RNase $140,120,360 \mu \mathrm{g} / \mathrm{kg} / \mathrm{d}$ or vehicle-solution ( $\mathrm{NaCl} 0.9 \%, \mathrm{n}=10$ each) by intraperitoneal injection 30 minutes an $12 \mathrm{~h}$ after brain insult. Treatment significantly reduced brain lesion volume in all treatment groups. With equal regime and amount of RNase1 120h after CCI (B) impact lesion volume revealed no persistent treatment success. 
Neurofunctional deficits were determined at 24h (C) and showed a dose dependent effect on outcome with low dose showing the best score but failing to reach level of significance. The motoric function (D) was severely affected after 24 hours and animals recovered mainly after 120 hours without significant difference between treatment and vehicle ( $n=10 /$ group). Data are presented as mean \pm standard deviation; $\mathrm{P}$ values are adjusted for multiple comparisons by Sidak correction.

A

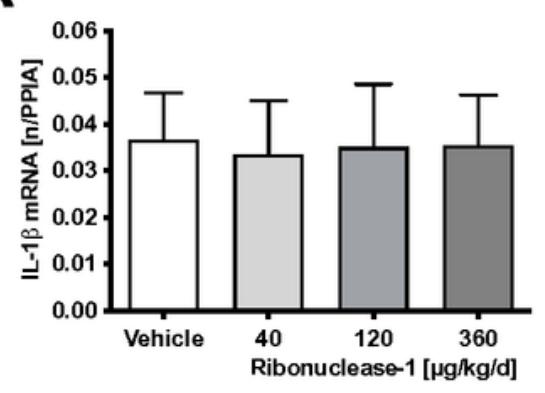

C

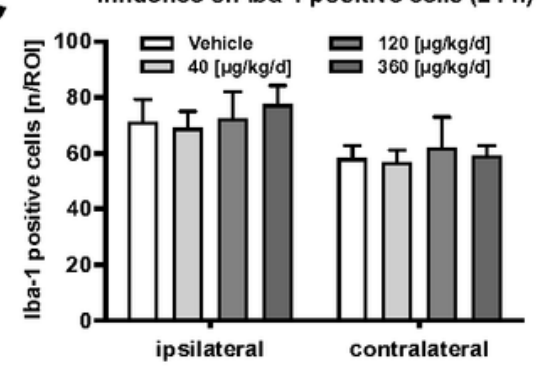

D

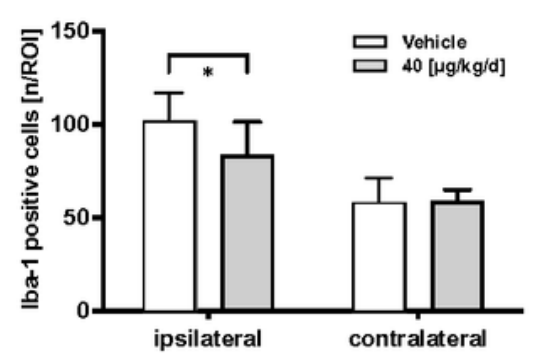

$\mathbf{E}$

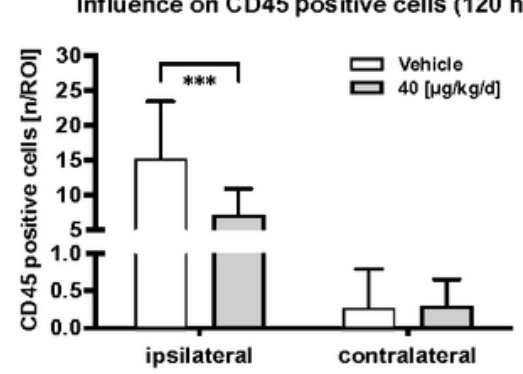

B
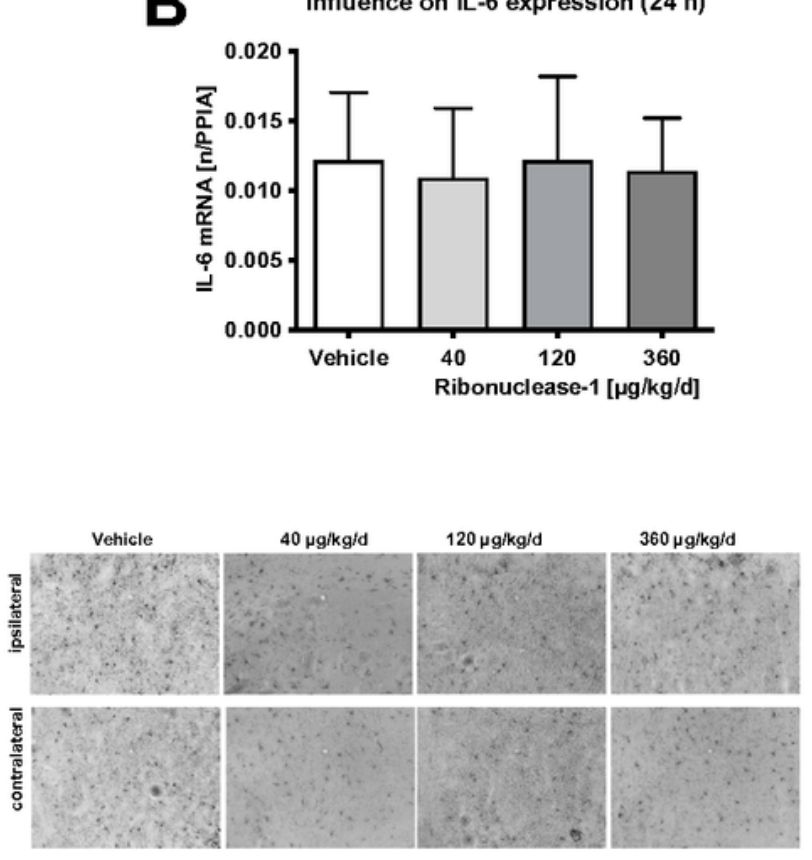

Vehicle
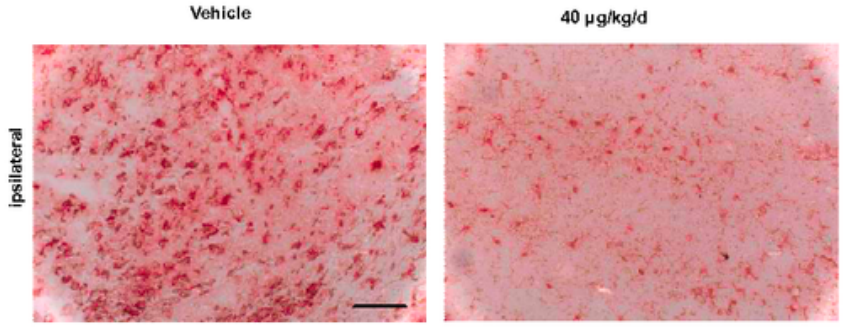

Vehicle

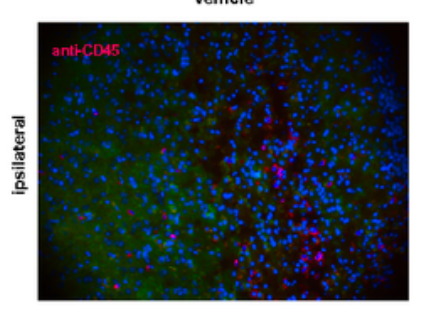

$40 \mu \mathrm{g} / \mathrm{kg} / \mathrm{d}$

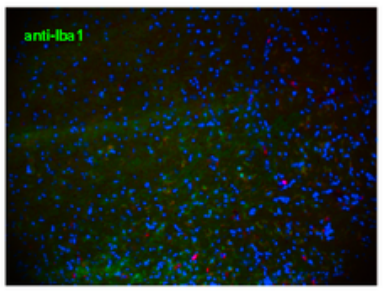

Figure 2 
Regulation of markers for inflammation Eexpression of inflammatory marker genes IL-1 $\beta$ (A) and IL- 6 (B) were quantified and showed no treatment dependent effect. As marker for microglia activation the number of Iba- 1 positive cells (C) were quantified and showed no difference between treatment $(n=10$ each) groups $24 \mathrm{~h}$ after $\mathrm{CCl}$. Contralateral number of Iba-1 positive cells remained identical $120 \mathrm{~h}$ after trauma. Pericontusional increase of Iba1- cells was significant lower in the RNase1 treated mice (D) linked to a significant decrease of CD45 positive cells (E) in the RNase group ( $n=10 /$ group) $120 \mathrm{~h}$ after $\mathrm{CCl}$. Data are presented as mean \pm standard deviation; $\mathrm{P}$ values are adjusted for multiple comparisons by Sidak correction. 
A

Influence on brain edema formation (24 h)

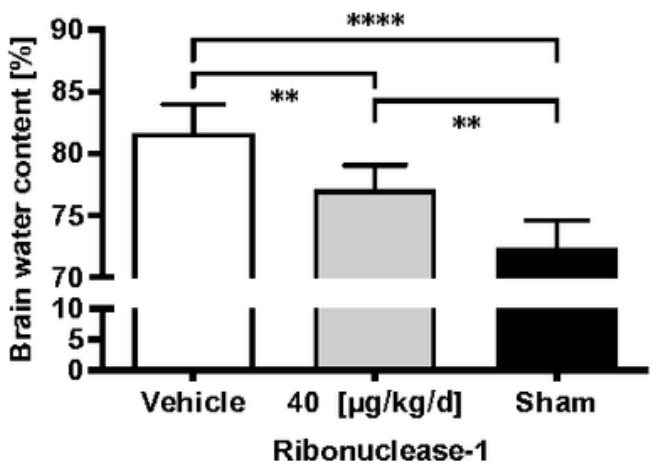

I Influence on blood-brain-barrier (24 h)

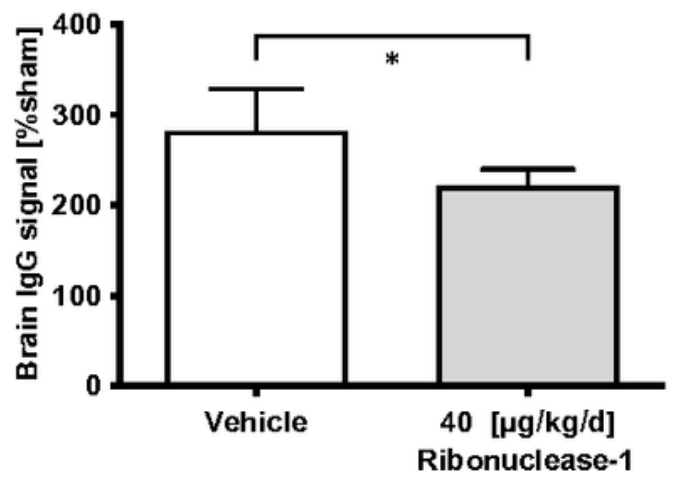

ipsi contra

Sham

Vehicle

$40 \mu \mathrm{g} / \mathrm{kg} / \mathrm{d}$
Influence on Claudin 5 expression (24 h)

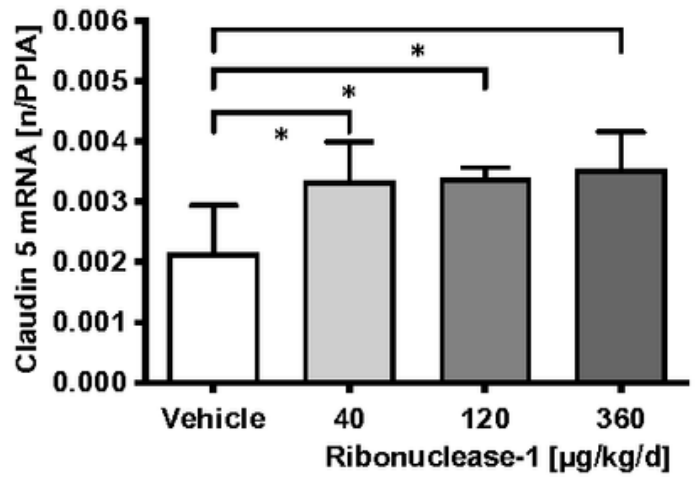

D Influence on ZO-1 expression (24 h)

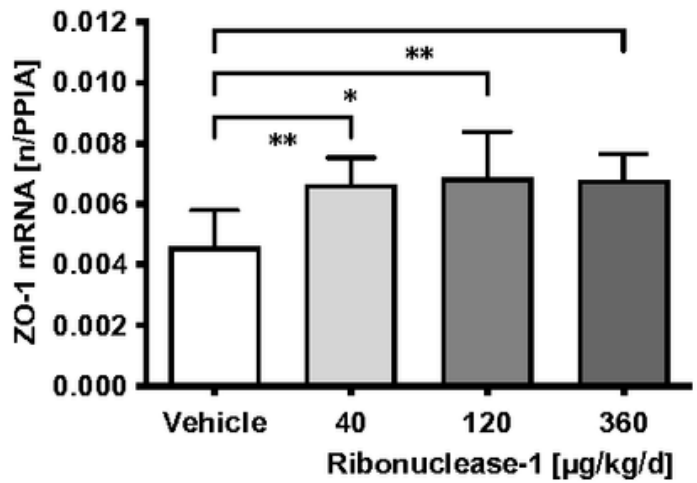

E Influence on ZO-2 expression (24 h)

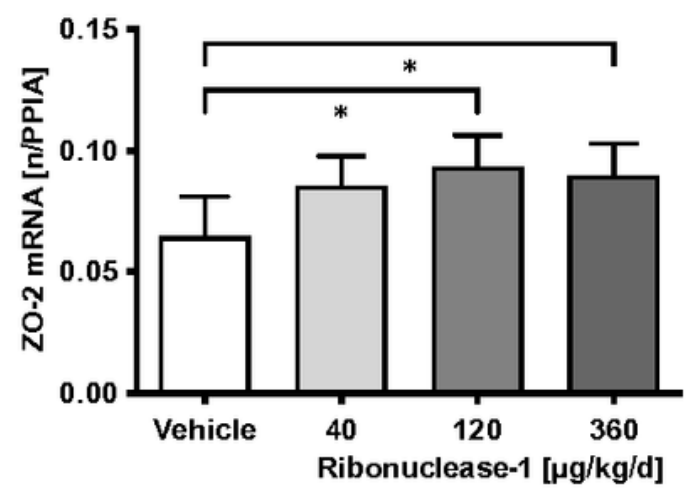

Figure 3

Influence on cerebral edema und regulation of tight junctions mRNA Brain water content determined $24 \mathrm{~h}$ after $\mathrm{CCl}(\mathrm{A})$ was significantly lower with $40 \mu \mathrm{g} / \mathrm{kg} / \mathrm{d}$ RNase1 compared to vehicle solution ( $\mathrm{n}=10$ each). Integrity of the blood brain barrier was investigated $24 \mathrm{~h}$ after lesion by quantification of immunoglobulin $\mathrm{G}(\mathrm{IgG})$ extravasation (B), which was significantly lower in RNase treated animals ( $\mathrm{n}=10$ each). 24h after insult mRNA expression of claudin 5 (C), ZO-1 (D) and ZO-2 (E) was quantified to investigate the impact 
of RNase treatment on VEGF regulated tight junction protein and showed significantly higher values with RNase treatment ( $n=10$ each). Data are presented as mean \pm standard deviation; $P$ values are adjusted for multiple comparisons by Sidak correction.

\section{Study Design}

24 h: Nissl-, Iba1-Staining and mRNA processing, Neuro-Deficit-Score (NDS)

\section{Vehicle}

RNasel low dose (LD)

RNasel intermedaiate dose (MD)

RNasel high dose (HD)

24 h: Brain Water, IgG

RNase low dose (LD)

Sham
Vehicle

$$
\begin{array}{ll}
(2 * 0,9 \% \mathrm{NaCl}) & \mathrm{n}=10 \\
(2 * 20 \mu \mathrm{g} \text { RNase } 1 / \mathrm{kg}) & \mathrm{n}=10 \\
(2 * 60 \mu \mathrm{g} \text { RNase } / \mathrm{kg}) & \mathrm{n}=10 \\
(2 * 180 \mu \mathrm{g} \text { RNasel } / \mathrm{kg}) & \mathrm{n}=10
\end{array}
$$
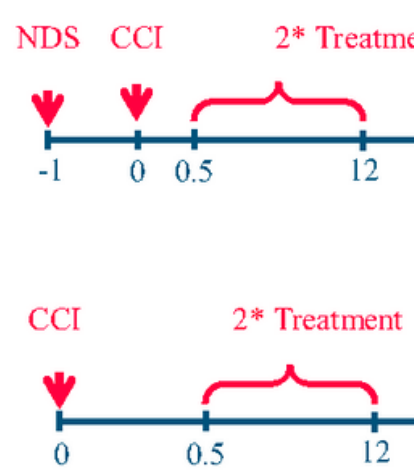

Brain tissue processing

120 h: Nissl-, Iba1- and CD45-Staining, Rota-Rod-Test (RR)

$$
\begin{array}{ll}
(2 * 0,9 \% \mathrm{NaCl}) & \mathrm{n}=10 \\
(2 * 20 \mu \mathrm{g} \text { RNase } 1 / \mathrm{kg}) & \mathrm{n}=10 \\
& \mathrm{n}=10
\end{array}
$$

Brain tissue processing

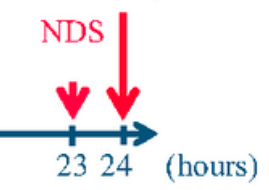

\section{Vehicle}

RNase low dose (LD)
$(2 * 0,9 \% \mathrm{NaCl})$

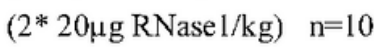

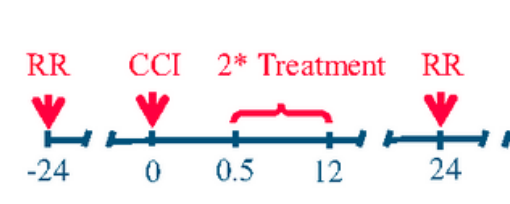

Brain tissue processing

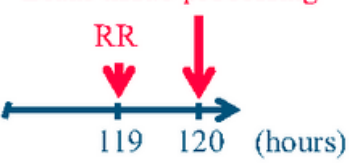

\section{Figure 4}

Study Design The study was performed with 90 male mice. Only the most effective (low) dose of RNase1 was used for subsequent studies of brain water accumulation, tissue IgG changes, and 120-hour survival. 